\title{
Neutrino oscillations in low density medium
}

\author{
A. N. Ioannisian ${ }^{a, b}$ and A. Yu. Smirnov ${ }^{c, d}$ \\ ${ }^{a}$ Yerevan Physics Institute, Alikhanian Br. 2, 375036 Yerevan, Armenia \\ ${ }^{b}$ Institute for Theoretical Physics and Modeling, 375036 Yerevan, Armenia \\ ${ }^{c}$ Department of Physics, Tokyo Metropolitan University, Hachioji, Tokyo 192-0397, Japan \\ ${ }^{d}$ ICTP, Strada Costiera 11, 34014 Trieste, Italy
}

\begin{abstract}
For the case of small matter effects: $V \ll \Delta m^{2} / 2 E$, where $V$ is the matter potential, we develop the perturbation theory using $\epsilon \equiv 2 \mathrm{VE} / \Delta \mathrm{m}^{2}$ as the expansion parameter. We derive simple and physically transparent formulas for the oscillation probabilities in the lowest order in $\epsilon$ which are valid for arbitrary density profile. The formulas can be applied for propagation of the solar and supernova neutrinos in matter of the Earth, substantially simplifying numerical calculations. Using these formulas we study sensitivity of the oscillation effects to structures of the density profile situated at different distances from the detector $d$. We show that for the mass-to-flavor state transitions, e.g., $\nu_{2} \rightarrow \nu_{e}$, the sensitivity is suppressed for remote structures: $d>l_{\nu} E / \Delta E$, where $l_{\nu}$ is the oscillation length and $\Delta E / E$ is the energy resolution of detector.
\end{abstract}

\section{Introduction}

For the LMA oscillation parameters the oscillations of solar and supernova (low energy) neutrinos inside the Earth occur in the weak matter effect regime. That is, the matter potential, $V$, is much smaller than the kinetic energy of the neutrino system:

$$
V \ll \frac{\Delta m^{2}}{2 E}
$$

where

$$
V(x)=\sqrt{2} G_{F} N_{e}(x),
$$

$G_{F}$ is the Fermi constant, $N_{e}(x)$ is the number density of the electrons, and $\Delta m^{2}=m_{2}^{2}-m_{1}^{2}$ is the mass squared difference.

In this case one can introduce a small parameter

$$
\epsilon(x)=\frac{2 E V(x)}{\Delta m^{2}} \ll 1
$$

and develop the perturbation theory in $\epsilon(x)$.

Neutrino oscillations in the weak matter effect regime have been discussed extensively before [1] - [20], in particular, for the solar and supernovae neutrinos propagating in the matter of the Earth. The previous work has been done mainly in the approximation of density profile which consists of several layers with constant density.

Here we derive general formula which is valid for arbitrary density profile provided that the condition (1) is satisfied. The formula is the generalization of our result in [20] obtained for thin layers of matter. 


\section{$2 \quad \epsilon$ - perturbation theory}

We will consider two active neutrino mixing

$$
\nu_{f}=U(\theta) \nu_{\text {mass }}
$$

where $\nu_{f} \equiv\left(\nu_{e}, \nu_{a}\right)^{T}, \nu_{\text {mass }} \equiv\left(\nu_{1}, \nu_{2}\right)^{T}$ are the flavor and mass states correspondingly, and

$$
U \equiv\left(\begin{array}{cc}
\cos \theta & \sin \theta \\
-\sin \theta & \cos \theta
\end{array}\right)
$$

is the mixing matrix in vacuum. In general $\nu_{a}$ is a combination of $\nu_{\mu}$ and $\nu_{\tau}$.

We will first find the evolution matrix for the mass eigenstates and then make projection onto the flavor states. Evolution of the mass states is given by the equation

$$
i \frac{d \nu_{m a s s}}{d x}=H(x) \nu_{m a s s}
$$

with the Hamiltonian

$$
H(x)=\left(\begin{array}{cc}
0 & 0 \\
0 & \frac{\Delta m^{2}}{2 E}
\end{array}\right)+U^{\dagger}\left(\begin{array}{cc}
V(x) & 0 \\
0 & 0
\end{array}\right) U .
$$

The Hamiltonian can be rewritten as

$$
H(x)=U^{\prime}(x)\left(\begin{array}{cc}
0 & 0 \\
0 & \Delta_{m}(x)
\end{array}\right) U^{\prime \dagger}(x),
$$

where

$$
\Delta_{m}(x) \equiv \frac{\Delta m^{2}}{2 E} \sqrt{(\cos 2 \theta-\epsilon(x))^{2}+\sin ^{2} 2 \theta}
$$

is the difference of energies of the neutrino eigenstates in matter $\nu_{m} \equiv\left(\nu_{1 m}, \nu_{2 m}\right)^{T}$, and $U^{\prime} \equiv$ $U\left(\theta^{\prime}\right)$ is the instantaneous mixing matrix of the mass states in matter with the angle $\theta^{\prime}(x)$ determined by

$$
\sin 2 \theta^{\prime}(x)=\frac{\epsilon(x) \sin 2 \theta}{\sqrt{(\cos 2 \theta-\epsilon(x))^{2}+\sin ^{2} 2 \theta}} .
$$

Using (9) the expression for the mixing angle of the mass states in matter, $\theta^{\prime}(x)$, can be rewritten as

$$
\sin 2 \theta^{\prime}=\frac{V \sin 2 \theta}{\Delta_{m}}=\epsilon \sin 2 \theta_{m} .
$$

In the last equality of Eq. (11) $\theta_{m}$ is the mixing angle of the flavor states in matter. Taking into account relations $\nu_{f}=U\left(\theta_{m}\right) \nu_{m}$, and $\nu_{\text {mass }}=U\left(\theta^{\prime}\right) \nu_{m}$, we find that $\nu_{f}=U_{m}\left(U^{\prime}\right)^{\dagger} \nu_{\text {mass }}$, and consequently, $U=U_{m} U^{\prime}$. Therefore

$$
\theta^{\prime}=\theta_{m}-\theta
$$

The angle $\theta^{\prime}$ is small: $\sin 2 \theta^{\prime} \leq \epsilon$, as it should be in the weak matter effect regime.

The formal solution of the equation (6), that is, the evolution matrix from the initial point $x_{0}$ to the final point $x_{f}$, can be written as

$$
S\left(x_{0} \rightarrow x_{f}\right)=T e^{-i \int_{x_{0}}^{x_{f}} H(x) d x}
$$


where $T$ means the chronological ordering. Let us divide a trajectory of neutrinos into $n$ equal parts (layers) of the size, $\Delta x$, so that $n=\left(x_{f}-x_{0}\right) / \Delta x$, and assume constant density inside each layer. Then for the evolution matrix we obtain

$$
S\left(x_{0} \rightarrow x_{f}\right)=e^{-i H\left(x_{n}\right) \Delta x} \cdot e^{-i H\left(x_{n-1}\right) \Delta x} \cdots e^{-i H\left(x_{j}\right) \Delta x} \cdots e^{-i H\left(x_{1}\right) \Delta x}
$$

$\left(x_{n} \equiv x_{f}\right)$. According to (8) it can be written as

$$
S\left(x_{0} \rightarrow x_{f}\right)=U_{n}^{\prime} D_{n} U_{n}^{\prime \dagger} \cdot U_{n-1}^{\prime} D_{n-1} U_{n-1}^{\prime \dagger} \cdots U_{j}^{\prime} D_{j} U_{j}^{\prime \dagger} \cdots U_{1}^{\prime} D_{1} U_{1}^{\prime \dagger}
$$

where

$$
D_{j} \equiv\left(\begin{array}{cc}
1 & 0 \\
0 & e^{i \phi_{j}^{m}}
\end{array}\right), \quad \phi_{j}^{m} \equiv \Delta_{m}\left(V_{j}\right) \Delta x
$$

is the evolution matrix of the matter eigenstates in $j$-th layer, $\phi_{j}^{m}$ is the relative phase between the matter eigenstates acquired in the layer $j$, and $V_{j}$ is the value of potential in the $j$-th layer.

The mixing matrix $U_{j}^{\prime}$ is given by

$$
U_{i}^{\prime}=\left(\begin{array}{cc}
\cos \theta_{j}^{\prime} & \sin \theta_{j}^{\prime} \\
-\sin \theta_{j}^{\prime} & \cos \theta_{j}^{\prime}
\end{array}\right)
$$

where $\theta_{j}$ is the mixing angle of the mass states in the layer $j$.

Structure of the expression (15) is rather transparent: it is the product of the blocks $\left(U_{j}^{\prime} D_{j} U_{j}^{\prime \dagger}\right)$ for all the layers. In each layer we first project the mass states onto the matter eigenstates $\nu_{m}$, then evolve the eigenstates and then project back to the mass states.

Using (16) and (17) we find expression for the $j$-th block:

$$
U_{j}^{\prime} D_{j} U_{j}^{\prime \dagger}=D_{j}+G_{j}
$$

where

$$
G_{j}=\left(e^{i \phi_{j}}-1\right)\left[\frac{1}{2} \sin 2 \theta_{j}^{\prime}\left(\begin{array}{ll}
0 & 1 \\
1 & 0
\end{array}\right)+\sin ^{2} \theta_{j}^{\prime}\left(\begin{array}{cc}
1 & 0 \\
0 & -1
\end{array}\right)\right] .
$$

Notice that $G_{j}=O(\epsilon)$, furthermore the second term in (19), being proportional to $\sin ^{2} \theta^{\prime}$, is of the order $\epsilon^{2}$.

Inserting expression (18) into (15) we find

$$
S\left(x_{0} \rightarrow x_{n}\right)=\left(D_{n}+G_{n}\right)\left(D_{n-1}+G_{n-1}\right) \cdots\left(D_{1}+G_{1}\right),
$$

and in the form of series in powers of $G=O(\epsilon)$ it can be written as

$$
\begin{aligned}
S_{\left(x_{0} \rightarrow x_{f}\right)} & =D_{n} D_{n-1} \cdots D_{1} \\
& +\sum_{j=1}^{n} D_{n} \cdots D_{i+1} G_{j} D_{j-1} \cdots D_{1} \\
& +\sum_{j=1}^{n} \sum_{k=1}^{j-1} D_{n} \cdots D_{j+1} G_{j} D_{j-1} \cdots D_{k+1} G_{k} D_{k-1} \cdots D_{1}+\cdots
\end{aligned}
$$


The products of $D_{j}$ which appear in this formula equal

$$
\Pi_{j=k \ldots n} D_{j}=\exp \left(i \sum_{j=k \ldots n} \phi_{j}^{m}\right)=\exp \left(i \sum_{j=k \ldots n} \Delta_{m}\left(V_{j}\right) \Delta x\right) .
$$

In the limit $n \rightarrow \infty$ and $\Delta x \rightarrow 0$ the sums are substituted by the integrals: $\sum \Delta x \rightarrow \int d x$. So that

$$
\prod_{j=k \ldots n} D_{j} \rightarrow \exp \left(i \phi_{x_{k} \rightarrow x_{n}}^{m}\right)
$$

where

$$
\phi_{x_{k} \rightarrow x_{n}}^{m} \equiv \int_{x_{k}}^{x_{n}} d x \Delta_{m}(x) .
$$

Furthermore,

$$
G_{j} \rightarrow i \Upsilon(x) d x
$$

where according to (19)

$$
\Upsilon=\frac{1}{2} \sin 2 \theta V(x)\left(\begin{array}{ll}
0 & 1 \\
1 & 0
\end{array}\right)+\Delta_{m}(x) \sin ^{2} \theta^{\prime}(x)\left(\begin{array}{cc}
1 & 0 \\
0 & -1
\end{array}\right) .
$$

Here we have taken into account the relation (11).

Substituting the sums by the integrals in Eq. (21) we find the S-matrix in terms of $\Upsilon$ :

$$
\begin{aligned}
S\left(x_{0} \rightarrow x_{f}\right) & =\left(\begin{array}{cc}
1 & 0 \\
0 & e^{i \phi_{x_{0} \rightarrow x_{f}}^{m}}
\end{array}\right)+i \int_{x_{0}}^{x_{f}} d x\left(\begin{array}{cc}
1 & 0 \\
0 & e^{i \phi_{x \rightarrow x_{f}}^{m}}
\end{array}\right) \Upsilon(x)\left(\begin{array}{cc}
1 & 0 \\
0 & e^{i \phi_{x_{0} \rightarrow x}^{m}}
\end{array}\right) \\
& -\int_{x_{0}}^{x_{f}} d x \int_{x_{0}}^{x} d y\left(\begin{array}{cc}
1 & 0 \\
0 & e^{i \phi_{x \rightarrow x_{f}}^{m}}
\end{array}\right) \Upsilon(x)\left(\begin{array}{cc}
1 & 0 \\
0 & e^{i \phi_{y \rightarrow x}^{m}}
\end{array}\right) \Upsilon(y)\left(\begin{array}{cc}
1 & 0 \\
0 & e^{i \phi_{x_{0}}^{m} \rightarrow y}
\end{array}\right)+\cdots
\end{aligned}
$$

Essentially we perform an expansion of the $S$-matrix in powers of $\Upsilon$.

Let us take the zero and the first order terms in $\Upsilon$ in (27) which correspond to the zero and first order terms in $\epsilon$. We find

$$
S\left(x_{0} \rightarrow x_{f}\right)=\left(\begin{array}{cc}
1 & 0 \\
0 & e^{i \phi_{x_{0} \rightarrow x_{f}}^{m}}
\end{array}\right)+i \frac{\sin 2 \theta}{2} \int_{x_{0}}^{x_{f}} d x V(x)\left(\begin{array}{cc}
0 & e^{i \phi_{x_{0} \rightarrow x}^{m}} \\
e^{i \phi_{x \rightarrow x_{f}}^{m}} & 0
\end{array}\right) .
$$

An interesting feature of the formula (28) which leads to important consequences (see sec. 4 ) is that in the second term the 12-element is determined by the phase acquired from the initial point $x_{0}$ to a given point $x$, whereas the 12-element depends on the phase from a given point $x$ to the final point of evolution $x_{f}$.

The key feature of the method is that we calculate the adiabatic phase exactly, whereas the mixing and the amplitude of oscillations are found approximately using the perturbation in $\epsilon$.

Notice that the second term in $\Upsilon$ (26) (of the order $\epsilon^{2}$ ) is proportional to the diagonal matrix. The 11-element of this term does not contain oscillatory factor and therefore, being inserted in (27), produces the contribution to the $S$ - matrix which is proportional to the length of the trajectory. It can be shown that this large contribution is canceled precisely by the contribution from the term of the second order in $\Upsilon$ (second line in Eq. (27)). 


\section{Probabilities of neutrino conversion}

Using the evolution matrix in the mass states basis (28) we can calculate the amplitudes and probabilities of different transitions. The evolution matrix in the flavor basis, $S_{f}$, can be obtained immediately:

$$
S_{f}=U S U^{\dagger}
$$

The evolution matrix from the mass states to the flavor states, $S_{f m}$, is then

$$
S_{f m}=U S
$$

and $U$ is the vacuum mixing matrix (5).

Let us consider the most important examples. The amplitude of the mass-to-flavor transition, $\nu_{i} \rightarrow \nu_{\alpha}$, on the way from $x_{0}$ to $x_{f}$ is given by

$$
A_{\nu_{i} \rightarrow \nu_{\alpha}}=U_{\alpha j} S_{j i}
$$

Inserting the matrix (28) in this expression we find the amplitude of the $\nu_{1} \rightarrow \nu_{e}$ oscillations in the first order in $V$ :

$$
A_{\nu_{1} \rightarrow \nu_{e}}=\cos \theta+\frac{i}{2} \sin 2 \theta \sin \theta \int_{x_{0}}^{x_{f}} d x V(x) e^{i \phi_{x \rightarrow x_{f}}^{m}} .
$$

Then the probability of the $\nu_{1} \rightarrow \nu_{e}$ oscillations equals

$$
P_{\nu_{1} \rightarrow \nu_{e}}=\cos ^{2} \theta-\frac{1}{2} \sin ^{2} 2 \theta \int_{x_{0}}^{x_{f}} d x V(x) \sin \phi_{x \rightarrow x_{f}}^{m} .
$$

The probability of $\nu_{2} \rightarrow \nu_{e}$ transition relevant for the solar neutrino oscillations in the Earth can be obtained immediately from the unitarity condition:

$$
P_{\nu_{2} \rightarrow \nu_{e}}=\sin ^{2} \theta+\frac{1}{2} \sin ^{2} 2 \theta \int_{x_{0}}^{x_{f}} d x V(x) \sin \phi_{x \rightarrow x_{f}}^{m} .
$$

Then the regeneration parameter defined as $f_{r e g} \equiv P_{\nu_{2} \rightarrow \nu_{e}}-\sin ^{2} \theta$ (see, e.g., [10]) equals

$$
f_{\text {reg }}=\frac{1}{2} \sin ^{2} 2 \theta \int_{x_{0}}^{x_{f}} d x V(x) \sin \phi_{x \rightarrow x_{f}}^{m}
$$

Using expression for the phase $\phi_{x \rightarrow x_{f}}^{m}(24)$ we obtain explicitly

$$
f_{r e g}=\frac{1}{2} \sin ^{2} 2 \theta \int_{x_{0}}^{x_{f}} d x V(x) \sin \int_{x}^{x_{f}} d y \frac{\Delta m^{2}}{2 E} \sqrt{\left(\cos 2 \theta-\frac{2 V(y) E}{\Delta m^{2}}\right)^{2}+\sin ^{2} 2 \theta}
$$

Let us underline that these expressions are valid for arbitrary density profile provided that the condition (1) is fulfilled.

The terms which contain $V$ are implicitly of the order $\epsilon$. Indeed, noting that the phase

$\phi_{x \rightarrow x_{f}}$ is proportional to $\Delta m^{2} / 2 E$ and changing the integration variable $x \rightarrow x \Delta m^{2} / 2 E$, one obtains the factor $\epsilon(x)$. 
It is straightforward to show that the obtained formulas reproduce the well know results for particular density distributions. Thus, for one layer with constant potential the Eq. (35) gives immediately:

$$
f_{r e g}=\epsilon \sin ^{2} 2 \theta \sin ^{2} \frac{\pi L}{l_{m}}
$$

where $l_{m}=2 \pi / \Delta_{m}$ is the oscillation length in matter. For the profile with $n$ symmetric shells we obtain performing explicit integration in (35)

$$
f_{r e g}=\frac{2 E}{\Delta m^{2}} \sin ^{2} 2 \theta \sin \Phi_{0} \sum_{i=0}^{n-1} \Delta V_{i} \sin \Phi_{i},
$$

where $\Phi_{0}$ is the total phase acquired along the trajectory and $\Phi_{i}$ is the phase acquired inside external radius of a given shell, $\Delta V_{i}$ is the jump of potential at border of the $i$-th shell [19]. In general, the formulas can be obtained from the adiabatic perturbation theory, performing integration by parts in Eq. (18) of [19]. In the lowest order in $\epsilon$ the adiabatic perturbation theory and the $\epsilon$-perturbation theory coincide.

\section{Sensitivity of oscillations to structures of the density profile}

The formulas obtained in section 3 can be used to estimate a sensitivity of the oscillation effects to various structures of density profile.

Suppose that there is some structure in the density profile (or $V$ ) in the point $x$. Then according to (33), (34) or (35) this feature will be integrated with $\sin \phi_{x \rightarrow x_{f}}$ - the periodic function whose phase is acquired from the structure position to the detector: $d \equiv x_{f}-x$. The larger the distance and therefore the phase, the stronger averaging effect is expected. So that the effect of remote structures of the profile on the mass-to-flavor oscillation probabilities is suppressed.

To quantify this sensitivity let us introduce the energy resolution function $f\left(E^{\prime}, E\right)$ and perform averaging of the probability folded with $f$ :

$$
\bar{P}_{\nu_{1} \rightarrow \nu_{e}}=\int d E^{\prime} f\left(E^{\prime}, E\right) P_{\nu_{1} \rightarrow \nu_{e}} .
$$

It is convenient to parameterize the effect of integration introducing the attenuation factor $F(d)$ in the probability as

$$
\bar{P}_{\nu_{1} \rightarrow \nu_{e}}=\cos ^{2} \theta-\frac{1}{2} \sin ^{2} 2 \theta \int_{x_{0}}^{x_{f}} d x V(x) F\left(x_{f}-x\right) \sin \phi_{x \rightarrow x_{f}}
$$

so that in the absence of averaging $F=1$.

Let us take for simplicity the box like resolution function $f\left(E^{\prime}, E\right)$ for which

$$
\bar{P}_{\nu_{1} \rightarrow \nu_{e}}=\frac{1}{\Delta E} \int_{E-\frac{\Delta E}{2}}^{E+\frac{\Delta E}{2}} d E P_{\nu_{1} \rightarrow \nu_{e}}
$$




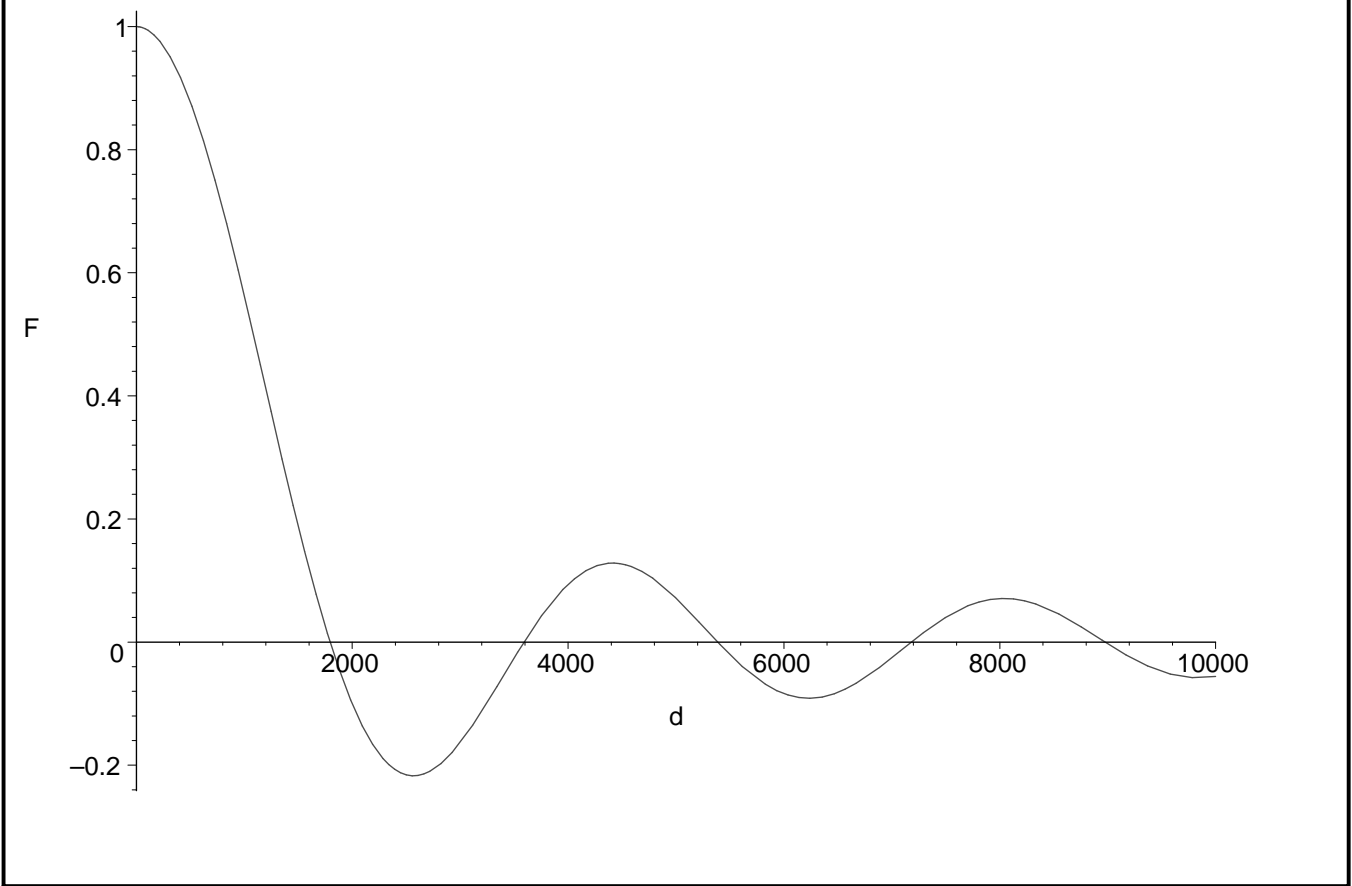

Figure 1: The attenuation factor $F$ as function of $d \equiv x_{f}-x$ for $E=10 \mathrm{MeV}, \Delta E=2 \mathrm{MeV}$, and $\Delta m^{2}=7 \cdot 10^{-5} \mathrm{eV}^{2}$.

Then assuming that $\Delta E \ll E$ and making use of the approximation $\Delta_{m} \simeq \Delta m^{2}[1-\epsilon \cos 2 \theta] / 2 E$ we find

$$
F(d)=\frac{1}{Q(d)} \sin Q(d), \quad Q(d) \equiv \frac{\pi d \Delta E}{l_{\nu} E},
$$

where $l_{\nu} \approx l_{m}$ is the oscillation length (see Fig. 1).

As follows from the Fig. 1, the factor $F(d)$ is decreasing function of the distance from detector. The decrease of $F$ means that contributions from the large distances to the integral (40) are suppressed. According to Fig. 1 which corresponds to $\Delta E / E=0.2$, the effect of structures at distances above $1500 \mathrm{~km}$ are attenuated by $F$ at least by factor 5 in comparison with those situated near the detector. Correspondingly, the sensitivity to remote structures is much weaker.

The larger $\Delta E$, the smaller the width of the first peak of $F(d)$. As follows from (42), the width and therefore the region of unsuppressed contributions due averaging are given by

$$
d<l_{\nu} \frac{E}{\Delta E}
$$


This attenuation effect allows us to explain some features of the zenith angle distribution of the regeneration factor. No significant enhancement of the regeneration has been found in numerical calculations for the trajectories which cross the core of the Earth in comparison with the trajectories crossing the mantle only, in spite of the fact that density of the core, and therefore $\epsilon$, are 2 - 3 times larger (see e.g. [10], [11], [13]). The explanation is straightforward: the border of the core is at about $D=3000$ from the surface of the Earth. Therefore, according to Fig. 1 the effect of the structures from such a distance is suppressed by the factor $3-5$ which compensate the increase of $\epsilon$. On the other hand, the effect of even small structures near the surface of the Earth can be substantial.

The conclusions we made so far are valid for the oscillations of the mass states to the flavor states. The situation is different for the inverse - flavor-to-mass states transitions. Indeed, for $\nu_{e} \rightarrow \nu_{1}$ the probability $P_{\nu_{e} \rightarrow \nu_{1}}$ has similar expression as in (33) but with phase acquired on the way from the initial point $x_{0}$ to the position of the structure $x: \phi_{x_{0} \rightarrow x}$. Therefore with the flavor-to-mass transition probability one would probe structures at the opposite (to the detector) side of the Earth. This general consideration is in agreement with our results for thin layers of matter [20].

For the flavor to flavor transition we obtain the probability

$$
P_{\nu_{e} \rightarrow \nu_{e}}=1-\sin ^{2} 2 \theta \sin ^{2} \frac{\phi_{x_{0} \rightarrow x_{f}}^{m}}{2}-\frac{1}{2} \sin ^{2} 2 \theta \cos 2 \theta \int_{x_{0}}^{x_{f}} d x V(x)\left[\sin \phi_{x_{0} \rightarrow x}^{m}+\sin \phi_{x \rightarrow x_{f}}^{m}\right]
$$

which is sensitive to structures near the surface from the both sides of the Earth.

The detailed analysis of the averaging effects and other applications of the obtained results will be given elsewhere [21].

\section{Conclusion}

We have derived expressions for the oscillation probabilities in matter with arbitrary density profile in the weak matter effect regime: $V \ll \Delta m^{2} / 2 E$. An accuracy of these expressions (33, $34,35)$ is determined by the parameter $\epsilon$.

The results can be applied for the solar and supernova (low energy) neutrinos crossing the Earth. They substantially simplify numerical calculations of the oscillation effects.

The obtained formulas reproduce the known probabilities for particular density distributions (one layer with constant density, several layers, etc.).

The formulas have very simple structure and can be used efficiently for analysis of various effects. In particular, the sensitivity of the oscillation probabilities to various structures of the density profile can be easily evaluated. We have found that the mass-to-flavor transition probabilities are sensitive to structures situated close enough to detector. Effect of the remote structures is attenuated. The distance which can be viewed by a detector is determined by the oscillation length divided by the energy resolution of the detector: $l_{\nu} E / \Delta E$. These results can be used in future in the oscillation tomography of the Earth.

\section{Acknowledgment}

The work of A.N.I. was partially supported by SCOPES grant 7AMPJ062161. 


\section{References}

[1] L. Wolfenstein, Phys. Rev. D 17, 2369 (1978); L. Wolfenstein, in "Neutrino-78", Purdue Univ. C3, (1978).

[2] S. P. Mikheyev and A. Yu. Smirnov, '86 Massive Neutrinos in Astrophysics and in Particle Physics, proceedings of the Sixth Moriond Workshop, edited by O. Fackler and J. Trân Thanh Vân (Editions Frontières, Gif-sur-Yvette, 1986), p. 355.

[3] J. Bouchez et. al., Z. Phys. C32, 499 (1986); M. Cribier et. al., Phys. Lett. B 182, 89 (1986); E. D. Carlson, Phys. Rev. D34, 1454 (1986).

[4] A.J. Baltz and J. Weneser, Phys. Rev. D35, 528 (1987).

[5] A. Dar et. al., Phys. Rev. D 35 (1987) 3607; S. P. Mikheyev and A. Yu. Smirnov, Sov. Phys. Usp. 30 (1987) 759; L. Cherry and K. Lande, Phys. Rev D 363571 (1987); S. Hiroi, H. Sakuma, T. Yanagida, M. Yoshimura, Phys. Lett. B198 403, (1987) and Prog. Theor. Phys. 78 1428, (1987); A. J. Baltz and J. Weneser, Phys. Rev. D37, 3364 (1988); M. Spiro and D. Vignaud, Phys. Lett. B 242297 (1990).

[6] J.N. Bahcall, P.I. Krastev, Phys. Rev. C56: 2839 (1997).

[7] E. Lisi and D. Montanino, Phys. Rev. D56, 1792 (1997).

[8] E.K. Akhmedov, Nucl. Phys. B538(1999)25.

[9] M. Maris, S.T. Petcov, Phys. Lett. B457:319, (1999).

[10] M. C. Gonzalez-Garcia, C. Pena-Garay, A. Yu. Smirnov, Phys. Rev. D63:113004, (2001).

[11] G. L. Fogli, E. Lisi, A. Marrone, D. Montanino and A. Palazzo, Phys. Rev. D 66, 053010 (2002).

[12] Lian-You Shan, Xin-Min Zhang, Phys. Rev. D65:113011, (2002).

[13] M. B. Smy et al., Super-Kamiokande Collaboration, Phys. Rev. D69(2004) 011104.

[14] C. Lunardini, A.Yu. Smirnov, Nucl. Phys. B616: 307,2001.

[15] V. D. Barger, D. Marfatia, K. Whisnant, B.P. Wood, Phys. Rev. D64:073009, (2001).

[16] A. S. Dighe, M. T. Keil, G. G. Raffelt JCAP 0306:006, (2003).

[17] M. Lindner, T. Ohlsson, R. Tomas, W. Winter, Astropart. Phys. 19 (2003) 755.

[18] A.S. Dighe, M. Kachelriess, G.G. Raffelt, R. Tomas, JCAP 0401:004, (2004).

[19] P.C. de Holanda, Wei Liao, A. Yu. Smirnov, hep-ph/0404042.

[20] A. N. Ioannisian, A. Yu. Smirnov, hep-ph/0201012.

[21] A. N. Ioannisian and A. Yu. Smirnov, in preparation. 